\title{
VALOR DE LO LÚDICO EN LOS PROGRAMAS EDUCATIVOS CON ADULTOS MAYORES DE LA ISLA DE LA JUVENTUD
}

\author{
THE VALUE OF PLAYFUL EDUCATIONAL PROGRAMS WITH ELDERS OF THE \\ "ISLA DE LA JUVENTUD"
}

\section{O VALOR DO LÚDICO NOS PROGRAMAS EDUCATIVOS COM IDOSOS DA “ISLA DE LA JUVENTUD"}

Clara Lig Long Rangel ${ }^{\star}$

\begin{abstract}
Resumen: En general, lo lúdico, no se circunscribe al juego, abarca un amplio círculo de acciones humanas. En ellas priman el movimiento de aquí para allá, la espontaneidad, libertad, jolgorio, el entretenimiento y que proporcionan a las personas alegría y satisfacción. Son abundantes los trabajos referidos al juego en la etapa infantil, pero, es muy limitada todavía, la bibliografía referida al juego asociado a la vejez, aun cuando la magnitud y celeridad del envejecimiento demográfico a nivel mundial ha promovido el estudio y profundización en las diferentes perspectivas de esa última etapa del curso vital. Con el objetivo de promover la reflexión acerca del valor de lo lúdico en los programas educativos con personas mayores, en este trabajo se argumenta su importancia psicosociocultural, desde la perspectiva del desarrollo humano, sustentada en el enfoque histórico cultural y en aportaciones de la Educación Popular y, se presentan los resultados del proceso de investigación acción ejecutado durante el período 2005-2010 en la Facultad de Ciencias Médicas de la Isla de la Juventud, en el que se utilizaron diferentes dispositivos culturales en ambiente lúdico para estimular el autodesarrollo de gerontes; dichos dispositivos fueron validados mediante la opinión de usuarios.
\end{abstract}

Palabras claves: Juego. Diversión. Gerontes. Autodesarrollo. Alegría. Satisfacción.

\begin{abstract}
In general, the playful, not limited to play, includes a wide circle of human actions. In them press the movement from here to there, spontaneity, freedom, the mess, the entertainment, that give people joy and satisfaction. There are abundant works referred to the game in step children, but is very limited the bibliography referred to when playing is associated with old age, even when the magnitude and speed of ageing populations worldwide has promoted the study and the deepening in the different perspectives of the last stage of the vital course. With the aim of promoting reflection on the value of playful educational programs with the elderly, in this paper it is argued its psycho-sociocultural importance, from the perspective of human development, sustained in the historic cultural approach and the contributions of Popular Education, and if they present the results of the research-action process executed during the period 2005-2010 at the College of Medical Sciences of the Isla de la Juventud, in which different cultural devices were used in playful environment to stimulate the self-development of gerontes; these devices were validated by the opinion of users.
\end{abstract}

Keywords: : Game. Fun. Self-development. Joy. Satisfaction.

\footnotetext{
* Profesora Doutora. Coordenadora da Cátedra de Estudios sobre Adultez Mayor (CEAM) na Universidad de Ciencias Médicas de la Habana. Filial Isla de la Juventud em Cuba. E-mail: claraliglong@infomed.sld.cu
} 
Resumo: Em geral, o lúdico, não se circunscreve ao brincar, abarca um amplo círculo de ações humanas. Nelas primam o movimento daqui para lá, a espontaneidade, a liberdade, a bagunça, o entretenimento, que fornecem às pessoas alegria e satisfação. São abundantes os trabalhos referidos ao jogo na etapa infantil, mas, é muito limitada ainda a bibliografia referida ao brincar associado à velhice, mesmo quando a magnitude e celeridade do envelhecimento demográfico a nível mundial tenha promovido o estudo e o aprofundizamento nas diferentes perspectivas dessa última etapa do curso vital. Com o objetivo de promover a reflexão sobre o valor do lúdico nos programas educativos com idosos, neste trabalho argumenta-se sua importancia psico-socio-cultural, desde a perspectiva do desenvolvimento humano, sustentada no enfoque histórico cultural e nos aportes da Educação Popular, e se apresentam os resultados do processo de pesquisa-ação executado durante o período 2005-2010 na Faculdade de Ciências Médicas da Isla de la Juventud, no qual utilizaram-se diferentes dispositivos culturais em ambiente lúdico para estimular o auto-desenvolvimento de gerontes; os ditos dispositivos foram validados mediante a opinião de usuários.

Palavras-Chave: Jogo. Diversão. Gerontes. Auto-desenvolvimento. Alegria. Satisfação.

\section{Introducción}

El vocablo juego, utilizado tanto en sentido directo como figurado, posee una significación diferente en distintas culturas. Para los antiguos griegos figuraba la acción de los niños de hacer chiquilladas; para los hebreos se correspondía con chiste y de risa. Ludo, significaba para los romanos alegría, y para los antiguos germanos movimiento ligero, suave semejante al balanceo de un péndulo, que produce una gran satisfacción. (ELKONIN, 1984, p. 14-15). En la locución latina, se asocia a diversión, recreación, travesuras, esparcimiento e incluye adivinanzas y acertijos, prestidigitación (juego de manos), retruécanos (juego de palabras), ejercicios físicos, de equilibrio y con instrumentos. En síntesis, el juego, abarca un amplio círculo de acciones humanas -no tendientes a un trabajo difícilen las que priman el movimiento de aquí para allá, la espontaneidad, libertad, jolgorio y el entretenimiento, que proporcionan a las personas alegría y satisfacción.

Mucho antes de que los filósofos, pedagogos y psicólogos, convirtieran el juego en el objeto de las investigaciones científicas, era utilizado como un medio de educación de los niños, por su naturaleza social, ello responde, a la necesidad de comunicación que aparece tempranamente y que deviene tendencia a la vida en común con los otros. Son abundantes los trabajos referidos al juego en la etapa infantil, expresa ElKonin (1984) que en 1933, Lev Semiovich Vigotski con la amplitud y profundidad característicos, desarrolló este tema y lo presentó en el Instituto Pedagógico Hertzen, de Leningrado, como central para la compresión del desarrollo psíquico en la edad preescolar.

Sin embargo, es limitada la bibliografía referida al juego asociado a las personas mayores, aun cuando la magnitud y celeridad del envejecimiento demográfico a nivel mundial ha estimulado el estudio y profundización en las diferentes perspectivas del envejecimiento y la vejez. De hecho los estudios referidos a esta etapa del curso vital todavía constituyen un reto para las ciencias, en especial para la Gerontología, Psicogerontología y la Gerontagogía ramas de la Psicología y la Pedagogía a ella vinculadas- en las que se requieren esfuerzos teóricos para su abordaje desde una posición auténtica del desarrollo humano.

Con el objetivo de promover la reflexión acerca del valor de lo lúdico en los 
programas educativos con personas mayores, en este trabajo se argumenta su importancia psicosociocultural, desde la perspectiva del desarrollo humano, sustentada en el enfoque histórico cultural y en aportaciones de la Educación Popular y, se presentan los resultados de la investigación cualitativa, ejecutada mediante la metodología investigación-acción durante el período 20052010 en la Filial de Ciencias Médicas de la Isla de la Juventud, en el que se utilizó el capital cultural de los gerontes en ambiente lúdico para estimular el autodesarrollo de los participantes en los programas educativos; dichos dispositivos fueron validados mediante la opinión de usuarios.

\section{Desarrollo}

Se reconoce que la educabilidad es una cualidad inherente a los seres humanos. $\mathrm{Y}$ aunque tradicionalmente la educación, se relacionaba con procesos, prácticas, intenciones, condiciones y sujetos vinculados a determinadas instituciones educacionales; con la formación escolar, profesional o para el trabajo, ya hoy se habla de la educación para y a lo largo de toda la vida.

A propósito, se considera que en la adultez mayor o vejez, la educación, como expresan Méndez de Paraco y Corrales Mejías citadas por Clara Lig Long Rangel (2014) se refiere como en cualquier etapa de la vida a un proceso continuo del desarrollo armónico y dinámico en el ser humano del conjunto de sus potencialidades afectivas, morales, intelectuales, físicas y espirituales; desarrollo del sentido de autonomía, de la responsabilidad, de la decisión, de los valores humanos y de la felicidad de la persona, por lo que debe asumirse como una instrumento de empoderamiento y validación de la condición de ser humano, que fomenta que las mismas personas mayores sean protagonistas de su realidad y transformación, de su propio desarrollo.

No obstante, es inadmisible pretender educar a las personas mayores desconociendo sus peculiaridades, como se hace los niños, adolescentes y jóvenes. A diferencia de aquellos, los/las gerontes, son conscientes de sus necesidades educativas, han adquirido a través de sus años de vida conocimientos, experiencias sociales y laborales, por les posibilitan medir los costos de su aprendizaje, ya sea en términos de tiempo, dinero o de oportunidades perdidas. Son maduros para razonar, seleccionar y determinar por decisión propia si buscan o no medios para educarse, para qué y en qué formas.

Como grupo de aprendizajes, los/ las educandos mayores (geroeducandos) forman grupos heterogéneos, en edad, sexo, historias de vida, intereses, experiencias, profesiones y oficios, entre otros aspectos sociodemográficos; tienen objetivos claros y concretos, elegidos y valorizados, sus fuentes del conocimiento son múltiples, se preocupan por el fracaso y pueden ser susceptibles a las críticas, a veces arrastran experiencias de aprendizajes frustrantes, aunque poseen mecanismos de compensación, gustan de aprovechar el tiempo, necesitan alternativas y variantes por su relativa capacidad de esfuerzo intelectual prolongado.

Por tales motivos, los programas educativos para y con los adultos mayores han de concebirse de manera típicamente diferente a las tradicionales. Han de ser personalizados y contextualizados y a la vez flexibles, atrayentes y provocadores pero contenidos, conquistadores no esclavizadores sino liberadores. Atributos que se pudieran ilustrar con un breve relato:

Cierta vez, durante el desarrollo de uno de los talleres del curso Cultura de paz y de 
Conflictos en la familia multigeneracional - el cual busca contribuir a visualizar, mediante caricaturas, cuán violentos somos o podemos ser, cuando en los vínculos interpersonales median relaciones de dominación- una adulta mayor pidió la palabra para leer un escrito: "una sonrisa cuesta poco y produce mucho. No empobrece a quien la da y enriquece a quien la recibe. Nadie hay tan rico que pueda vivir sin ella. Ni nadie tan pobre que no la merezca”. La inesperada lectura del texto, escrito por el reconocido cineasta Charles Chaplin estimuló la memoria de evocación. Al inició, todos reímos recordando algunos pasajes de sus extraordinarias películas, después le sucedieron otros no tan gratos, en los que hubo que "contener" al grupo para evitar el desborde emocional. Ese texto devino pretexto para dialogar acerca del mal trato que invade con relativa frecuencia los contextos familiares, laborales, escolares, entre otros espacios sociales. Para al cierre del taller quedó una puerta abierta para pensar en acciones que se pudieran hacer a corto o mediano plazo en y desde nuestros contextos de relaciones más próximos para prever y modificar las posturas violentas. En lo sucesivo, "llovieron" las iniciativas a la par que brotaban las confidencias y con ellas las relaciones de colaboración y cohesión grupal. Ese día el texto escrito traído por una asistente del taller fue el único material de apoyo utilizado, los que se habían previstos resultaron innecesarios para promover la participación, la introspección, la comunicación dialógica, la colaboración y la mirada al futuro. Ellas, porque eran todas mujeres, pasaron a ser protagonistas de su propio aprendizaje, avanzaron en su proceso de autodesarrollo.

\section{El adulto mayor como sujeto activo del proceso de autodesarrollo durante la ejecución de los programas educativos}

Cambiar siempre es un proceso difícil e imprescindible para el desarrollo. También en la adultez mayor es posible el desarrollo, como proceso único de autodesarrollo, en el que se forma y surge un nuevo tipo de estructura de la personalidad y su actividad; cambios psicológicos y sociales de conjunto que se producen por primera vez en cada edad, que tienen lugar mediante la interacción de unos sujeto con otros, solo es posible mediante la actividad y la comunicación en sus diferentes contextos de relaciones sociales y es resultado de un complejo sistema de influencias educativas, intencionadas o no, formales e informales.

A partir de esas consideraciones, para concebir el proceso de desarrollo personal (personológico y social) y lograr su estimulación durante la ejecución de los programas educativos, los educadores de adultos mayores, o sea, los geroeducadores o gerontagogos realizaron un diagnóstico participativo contextualizado individual y grupal e identificaron las potencialidades y limitaciones de cada geronte, su autopercepción de ellos y de su vida pasado y presente y su autoproyección del futuro que estiman como posible, según su contexto sociocultural. Además, consideraron otros aspectos emergentes en el diagnóstico: desconocimiento la etapa de la vida y de sus potencialidades individuales; pobre motivación e implicación en la elaboración de proyectos vitales; sentimientos de limitación y enfermedad; inseguridad e inhibición; poca reflexión cognitiva y metacognitiva; dificultades en la autoestima y de convivencia; necesidades de comunicación, socialización e intercambio y de reconocimiento social. 
A partir del conocimiento de la pluralidad de intereses, necesidades y características individuales, grupales esenciales y de los factores incidentes en las relaciones sociales de los gerontes, entre otros rasgos identitarios, singularizantes, y teniendo en cuenta que la forma en que se organizan las actividades concebidas en los programas educativos influye en la concientización de los adultos mayores de su progreso y desarrollo social; se buscó armonizar las acciones y evitar su contraposición, para favorecer que las personas mayores descubrieran los objetivos esenciales de su existencia y sintieran la necesidad de prepararse para el logro de los mismos.

Entonces, los gerontagogos organizaron procesos interactivos, sucesivos y simultáneos, en los que primaron el diálogo, la resignificación e intercambio reflexivo colaborativo de saberes, el trabajo grupal y se emplearon dispositivos culturales como herramientas pedagógicas diseñadas con antelación, para estimular la interactividad, y provocar la sinergia que podría movilizar en los/las gerontes cambios en el funcionamiento psicológico y social, en la dirección del autodesarrollo.

Con independencia de la edad, la actividad y la comunicación en unidad dialéctica, condicionante del desarrollo de los seres humanos, propició el intercambio consciente e inconsciente de ideas, actitudes, opiniones y vivencias afectivas posibilitó que las personas mayores se convirtieran en protagonistas, en sujetos activos, del proceso de autodesarrollo. Por otra parte, el clima psicogerontológico abierto y franco, de comunicación dialógica facilitó afloraran los interaprendizajes y con ello se fueron modificando su autoimagen, su autonomía y autodeterminación, y fueran cada vez más capaces de lograr su autotransformación psicosocial activa, constructiva y creadora, en íntima interrelación con el compromiso y la responsabilidad social, lo cual propició toda la gama de acciones y sentimientos humanos en su autoperfeccionamiento constante.

Se evidenció que es posible devolver el protagonismo a los adultos mayores, dándoles la oportunidad para participar y reaprender autónoma y conscientemente. Que los programas educativos promueven el autodesarrollo de los/las gerontes cuando su ejecución se dinamiza con la implementación de herramientas interactivas Se consideran como tales aquellos elementos del acervo cultural e histórico de un grupo etáreo, que utilizados -en este caso- como recursos gerontagógicos valorizan sus códigos de comunicación, tradiciones, valores, necesidades, sus potencialidades y las oportunidades de su contexto social más próximo. (RANGEL, 2012). Previamente identificadas en el diagnóstico participativo contextualizado, las herramientas propician la implementación de los programas en ambiente lúdico, es decir de juego, para provocar un aprendizaje gozoso.

No obstante la diversidad de expresiones distintivas de los cambios psicológicos y sociales propios del autodesarrollo en cada persona, es común a todos la presencia de la dimensión participativa, no solo como favorecedora de la calidad de las interacciones, sino como condición para que en la actividad y la comunicación con los otros se originen lo intersubjetivo o externo y lo intrasubjetivo o interno. Por otra parte, la heterogeneidad de experiencias culturales acumuladas por los gerontes a lo largo de sus historias de vidas, así como la participación, como posibilidad real de ser parte y tomar parte en el proceso de reaprendizaje para enfrentar los retos que impone el proceso de autodesarrollo, convocaban al empleo de las dinámicas interactivas, ya que logran: a) Contribuir a la recuperación de los saberes 
previos experienciales de los adultos mayores; b) introducir a los adultos mayores en una nueva trama de relaciones sociales que difieren de sus experiencias previas de socialización; c) la interdisciplinariedad en la organización de las actividades; d) la elaboración de propuestas flexibles, participativas, abiertas a sistemas y modelos de formación alternativos, a diferentes formas de organización de espacio, tiempo y estilos de aprendizaje; e) destacar las posibilidades de aplicación de las apropiaciones formativas en acciones socialmente responsables; f) impulsar la comunicación intergeneracional, favoreciendo la integración de las personas de los distintos grupos de edades.

De tal manera, los/las gerontes progresivamente lograron convertirse en sujetos activos de su propio desarrollo personal, de su autodesarrollo, debido a la viabilización los procesos de reaprendizaje para la reorganización de los recursos personales, en la dirección de los procesos de adaptación y búsqueda de soluciones a las situaciones dilemáticas vitales que ellos, como adultos mayores, debe afrontar en esta última etapa de sus vidas.

Fue notorio que el éxito, de los programas educativos con adultos mayores se favorece, en la medida en que la organización de sus actividades, sustentada en el conocimiento de las características individuales y grupales, en las expectativas y necesidades de sus participantes, propicia las mayores interacciones de cooperación, acuerdo, adaptación y asociación en las que el ambiente psicológicamente es gratificante. Por el contrario el éxito se entorpece cuando se promueven pocas interacciones, y cuando en las mismas muchas o pocas, priman la competencia, el desacuerdo, la oposición y la disociación y en consecuencia el clima o ambiente psicológico resulta tensionante.

\section{Lo lúdico en los programas educativos con adultos mayores de la Isla de la Juventud.}

Con el empleo de las dinámicas interactivas -los procedimientos y las herramienta interactivas- se introduce lo lúdico, se propicia el ambiente de juego, para que las interacciones de cooperación, y acuerdo, que difieren de las de competencia y desacuerdo, afiancen las relaciones interpersonales, motiven la participación, el intercambio de saberes, la ayuda mutua, ofrezcan a los adultos mayores la oportunidad de desinhibirse y protagonizar con independencia, conscientes y gozosamente su proceso de reaprendizaje en un entorno de alegría, de disfrute y satisfacción en el que pueden vivenciar los cambios en su autoestima, autopercepción y otros autorreferentes, y sentir que sí pueden.

Es necesario resaltar que el ambiente de juego, lo lúdico, tiene un propósito (COLECTIVO DE AUTORES, 2012, p. 168), no se trata de añadir algunos recursos o técnicas para erradicar la monotonía, el aburrimiento, la pasividad o para hacer más entretenida la ejecución de las actividades previstas en los programas, ni de organizar juegos para los grupos de personas mayores se cohesionen o solo se diviertan, en el peor de los casos. Se trata de algo más profundo y complejo. El uso en ambiente de juego de las dinámicas interactivas tiene el propósito de devolver a los adultos mayores -incorporados a un grupo de aprendizaje- el protagonismo y la palabra, estimular su conciencia crítica, la adaptación activa a la realidad de sus vejeces, la capacidad de reaprender para cambiar y de ser agentes de cambio, procesos necesarios para favorecer el autodesarrollo.

De ahí que la importancia de lo lúdico radica en la capacidad de generar condiciones 
gratificantes que enriquezcan el ser y estar de los sujetos mayores que están atravesando y son atravesados por los avatares del tiempo, haciendo uso del acervo cultural e histórico que ellos poseen. De favorecer la sinergia para que se den los procesos necesarios para el autodesarrollo, descargando las ansiedades, las tensiones, el estrés negativo; desbloqueando la iniciativa y la creatividad; mejorando la socialización y evitando el ostracismo interpersonal haciendo que broten el entusiasmo y el saber experiencial; brindando apoyo psicoafectivo, lenidando las posibles fricciones grupales, reforzando conductas positivas y modelando aquellas que superen los mitos y esteriotipos sociales, que obstaculizan que las/los gerontes sean percibidas/percibidos como personas activas en desarrollo.

Entre el capital cultural utilizado como herramientas pedagógicas para promover la interactividad en la Isla de la Juventud, durante la ejecución de los programas educativos con adultos mayores, se encuentran: las técnicas participativas; poemas, trabalenguas, refranes; cuentos y/o fábulas; dibujos y/o caricaturas; las canciones, chistes y/o situaciones jocosas; crucigramas y/o acrósticos; los juegos; las manualidades; paseos y/o excursiones; la pintura. Algunos ejemplos:

- Técnicas participativas: Son herramientas de trabajo en grupo, que se usan para animar, desinhibir o integrar a los participantes, estimulan la participación y hacen más sencillos determinados contenidos; propician los procesos de concientización individual y de grupo como resultado de la actividad externa e interna. Ejemplos:

1.- "Pueblo viejo o pueblo nuevo"

Objetivos: Favorecer la comprensión de los cambios como necesidad del desarrollo de los pueblos y de las personas que en él habitan. Estimular la reflexión en torno a la relación pasado, presente, futuro como parte del devenir histórico de la existencia humana.

Procedimiento: Se muestran fotos, dibujos o anécdotas escritas de la Cuba prerrevolucionaria referidas a adultos mayores, niños y/o mujeres abandonados a su suerte. Se promueve el intercambio a partir de un conjunto de preguntas, que sirvan para contrastar lo viejo y lo nuevo. Para finalizar se pregunta: ¿Es en pueblo nuevo todo perfecto?; Si pudieras construir el PUEBLO de sus sueños ¿qué cambiaría?

- Uso de poemas, trabalenguas y refranes: Modalidades del arte popular que movilizan los recursos personales, estimulan las definiciones, explicaciones y búsquedas necesarias en relación con las situaciones que se pretenden transformar. Ejemplos:

1.- "Décimas del tiempo tiempo" (fragmento) de Raúl Ferrer:

Objetivo: Promover la reflexión acerca de las posibilidades que se tienen en la adultez mayor para hacer cosas nuevas.

Me duele que pase el día /no porque me ponga viejo / ni por la tarde que dejo / ni por su melancolía.

Me duele esa paz vacía / cuando la puede llenar / con la fiesta de luchar / el trabajo, la visita, / y con la suerte infinita / de las cosas por crear.

2.- Refranes: Pensamiento sentencioso que en pocas palabras expresa un hecho de la experiencia que forma parte de a cultura popular; se utiliza con el objetivo de promover el diálogo acerca de las enseñanzas implícitas en el refranero popular y su posible aplicación en el ámbito comunitario, para mejorar la calidad 
de la convivencia. Ejemplos:1 “Más vale quedarse para vestir santos, que... casarse para desvestir borrachos”; 2 "El que nace para tamal, ... del cielo le caen las hojas"; entre otros; 3 "Camarón que se duerme se... lo lleva la corriente" / se lo comen los turistas.

Procedimientos: Los gerontes se agrupan por temas, a partir de un hilo conductor para que unas reflexiones den paso a otras y finalmente se pueda sugerir cómo afrontar la cambiante cotidianidad.

- Cuentos y/o fábulas. Relato breve que generalmente encierra una enseñanza.

1.- "La fábula del erizo”.

Objetivos: Estimular la tolerancia a partir de destacar la importancia de aceptar lo diferente. Reflexionar acerca de la necesidad de promover los mejores valores individuales y de grupo.

Procedimiento: Se lee la fábula y después se promueve el diálogo acerca de su contenido seguidamente se comenta su moraleja: La mejor relación no es aquella que une a personas perfectas, sino aquella en que cada individuo aprende a vivir con los defectos de los demás y admirar sus cualidades. El texto dice:

"Durante la Edad de Hielo, muchos animales murieron a causa del frío. Los erizos dándose cuenta de la situación, decidieron unirse en grupos. De esa manera se abrigarían y protegerían entre sí, pero las espinas de cada uno herían a los compañeros más cercanos, los que justo ofrecían más calor. Por lo tanto decidieron alejarse unos de otros y empezaron a morir congelados. Así que tuvieron que hacer una elección, o aceptaban las espinas de sus compañeros o desaparecían de la Tierra. Con sabiduría, decidieron volver juntos. De esa forma aprendieron a convivir con las pequeñas heridas que la relación con una persona muy cercana puede ocasionar, ya que lo más importante es el calor del otro. De esa forma pudieron sobrevivir."

- Paseos y excursiones: Recorridos de recreo realizados generalmente en tiempo libre.

1. Paseo "Bellezas de mi ciudad". Objetivos: Valorizar de lo propio construido por todos, conocer el contexto sociocultural y apreciar sus transformaciones para fomentar la integración social.

Después de conversar acerca de las construcciones en la Isla de la Juventud y sobre la participación de los adultos mayores en ese proceso, se realiza un recorrido por la ciudad para apreciar sus bellezas y valorar en qué participaron y aún pueden participar los mayores.

- Canciones: Productos musicales de notoria influencia en el organismo humano, como modificadores de la conducta individual y social, y de las funciones psíquicas. Ejemplos:

1.- Canción “Amanecer feliz" de la sección infantil de la Revista "Buenos Días” del canal “Tele Rebelde” de la televisión cubana y "Color esperanza” una invitación al optimismo y las ganas de vivir, no obstante los dificultades del día a día.

Objetivo: Descifrar la diversidad de mensaje de optimismo que transmite.

Procedimiento: Se escucha la grabación. Se circula entre los participantes el texto de la canción para que en grupos 
lo lean y analicen, y digan qué les pareció. Se repite la canción para que los que la canten los que desean. Se comparten los textos de las caniones para cantarlas en otros momentos.

- Manualidades: Actividad manual que abarca distintas modalidades; pintura, trabajo con barro; artesanía, confecciones, de fácil asimilación y realización. Ejemplos:

\section{1.- Papier marché}

Objetivo: Facilitar la autoexpresión y desarrollar la creatividad, mediante la confección de objetos para las áreas de juego de un círculo infantil u otras instituciones sociales.

Procedimientos: Se muestra la técnica. A continuación se organizan las acciones de forma secuencial, mediante la asignación se roles. Se dialoga acerca de la participación social.

- Dibujos y/o caricaturas: Expresiones gráficas que se presta a la lectura polisémica.

1.- Érase una vez...

Objetivos: Construir una historia colectiva a partir de una lámina. Reflexionar en torno al tema que propone la historia.

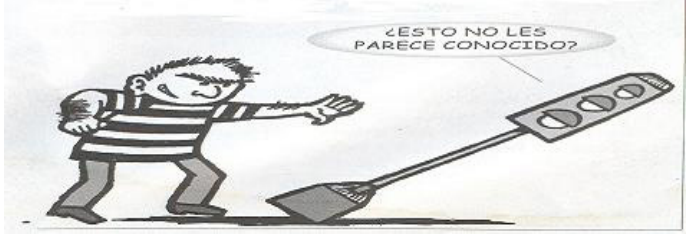

Procedimiento: Se utilizan preguntas para guiar la construcción: ¿Qué muestra la lámina?; ¿qué refleja la cara y postura de la persona?; ¿qué está haciendo esa persona? Posteriormente el equipo de constructores pide al grupo sus opiniones para los puntos de encuentros y de desencuentros.
- Juegos: Actividad en la que se combinan diversos procesos mentales y afectivos en la solución de problemas de distinta complejidad, en un ambiente de diversión. Ejemplos:

1.- Juego matemático.

Objetivo: Utilizar la actividad lúdica para favorecer los procesos del pensamiento.

Procedimiento: Se confeccionan varios tableros con distintos números. Se tiene un listado de informaciones que deben ser completas con un dato numérico, que es pronunciado por un participante cualquiera. En una bolsa se colocan las fichas correspondientes al orden de cada información en el listado. Cada vez que un dato numérico aparezca en su tablero la persona lo cubre con un botón o un círculo de cartón; la que primero llegue a cubrir su tablero, da por terminado, o sea cierra el juego, con la expresión o gesto que se le ocurra.

\begin{tabular}{c|c}
\hline $1^{*}$ & $*$ \\
\hline$*$ & $10^{*}$ \\
\hline $8^{*}$ & $15^{*}$ \\
\hline $2010^{*}$ & $30^{*}$ \\
\hline
\end{tabular}

Tablero de Rosa

\begin{tabular}{c|c}
\hline $1 *$ & $1959 *$ \\
\hline$*$ & $5 *$ \\
\hline $2 *$ & $15^{*}$ \\
\hline$*$ & $30 *$ \\
\hline
\end{tabular}

Tablero de Andrés

\begin{tabular}{c|c}
\hline $1 *$ & $10 *$ \\
\hline$*$ & $5 *$ \\
\hline $2 *$ & 23 \\
\hline $3 *$ & $*$
\end{tabular}

Tablero de Ernesto

Listado (colocado en orden de aparición de las fichas):

1) Mes y año en que triunfa la Revolución Cubana. (1, 1959)

3) Día y/o mes en que fueron excarcelados los Moncadistas del 
Presidio Modelo, de la antigua Isla de Pinos, hoy Isla de la Juventud $(15,5)$

2) Año pasado (2010)

6) Canal de televisión para ver la Mesa Redonda, en la Isla de la Juventud (8)

5) Día y mes en que nació la persona que más quieres (Cada uno cubrirá los dos cuadros dejados en blanco) Se aprovecha para la socialización de esa información.

9) Cantidad de adultos mayores que asisten a las actividades de la Cátedra de Estudios sobre Adultez Mayor (30)

4) Cantidad de viajes que da el katamarán los domingos, si no se rompe (2)

8) Dos por dos, más seis (10)

En este ejemplo el asterisco simboliza el boto que cubre el número en el tablero, se puede observar que Rosa es la que cierra el juego ya que cubrió la totalidad del tablero.

El tamaño de los tableros, así como la complejidad y contenido de la información que contenida en el listado, estarán en dependencia de los objetivos y particularidades del grupo de gerontes. Al finalizar el juego, se sugiere al grupo intercambien, como recuerdo una de las tarjetas confeccionadas por ellos durante la actividad de manualidades.

- Crucigramas y/o acrósticos: Textos con palabras escritas en un papel cuadriculado.

Objetivos: Ofrecer información útil referida a la adultez mayor. Estimular los procesos del pensamiento. Ejemplo: Un acróstico

\begin{tabular}{|c|c|c|c|c|c|c|}
\hline 1) $\mathrm{V}$ & I & 2) $\mathrm{V}$ & I & $\mathbf{R}$ & & \\
\hline & & A & & & & \\
\hline & & $\mathbf{L}$ & & & & \\
\hline 3) $\mathrm{S}$ & I & $\mathbf{E}$ & $\mathbf{M}$ & $\mathbf{P}$ & $\mathbf{R}$ & 1 \\
\hline
\end{tabular}

Procedimiento: En voz alta se leen las palabras para que sean colocadas de forma horizontal o vertical: 1.Vida, existencia; 2. Del verbo valer; 3. Adverbio de tiempo

- Pintura: Arte universal de la plástica que propicia canalizar sentimientos, expresar deseos, a la vez que ofrece información sobre gustos y necesidades.

1.- Pintura dactilar o pintura en agua.

Objetivos: Liberar tensiones emocionales y despertar vivencias agradables. Favorecer la cohesión del grupo mediante la confeccionar afiches para el local donde ellos trabajan.

Procedimientos: Se muestra la técnica. A continuación se organizan las acciones de forma secuencial, mediante la asignación de roles.

En síntesis, desde una visión psicosociocultural y gerontagógica, por su naturaleza, sociocultural e histórica, y su carácter familiar, lo lúdico en los programas educativos con adultos mayores incide favorablemente en su desarrollo personal pues promueven cambios en su funcionamiento psicosocial, ya que:

1) Refuerzan las autorreferencias positivas (autoestima entre otras). 2) Estimulan la convivencia respetuosa, la colaboración, sentimientos de respeto y aceptación a los otros, la comunicación intergeneracional. 3) Propician una posición activa ante el autodesarrollo. 4) Incrementan la percepción de utilidad, refuerzan las convicciones orientadas 
al cumplimiento del deber social y favorece la implicación personal. 5) Contribuyen a la recuperación y colectivización de los saberes previos experienciales mediante la activación de los procesos mentales (memoria y otros) y al despliegue de la inteligencia cristalizada. 6) Propician la interactividad, el trabajo grupal, la colaboración y la ayuda mutua, el interaprendizaje de forma amena, interesante, atractiva y efectiva. 7) Incorpora procedimientos de trabajo en grupos en los que se recuperan y aprovechan de forma óptima las posibilidades afectivas y cognitivas de todos. 8) Estimulan el proceso de recreación de la cultura, a través de la resignificación permanente del pasado y el presente para proyectar el futuro; de la reconfiguración de la percepción del mundo (interno y externo); de la dialéctica entre lo viejo y lo nuevo, lo semejante y lo diferente. 9) Contribuyen en la satisfacción de las necesidades superiores: -necesidades de seguridad: sentirse seguro y a salvo; -necesidades de pertenencia y de amor: de relacionarse con otros, ser aceptado; -de autorealización: para encontrar auto satisfacción y darse cuenta del potencial propio; -necesidades de autoestima: sentimiento de logro, ser competente, ganar aprobación y aceptación y ser reconocido por los demás. 10) Viabilizan la relación con los otros, propician la formación de grupos de ayuda mutua y la restructuración del funcionamiento psicosocial de los adultos mayores.

\section{Conclusiones}

En opinión de los/las gerontes participantes lo lúdico propició en ellos, de forma gradual, un estado emocional más estable y placentero; una autoestima positiva; mejoró la comunicación interpersonal; les ayudó en la evaluación diáfana del sentido de la vida y en la transferencia de experiencias; contribuyó a la apropiación de nuevos aportes de la dociencia y estimuló a la realización de nuevos proyectos. Descubrió los recursos personales - personológicos y sociales de cada adulto mayor, lo que posibilitó al colectivo de geroeducadores la planificación personalizada de acciones de orientación y asesoría dirigidas al geronte, a su familia y a la comunidad, de esta forma se ampliaron las posibilidades educativas del programa.

Los geroeducadores destacan que dada su propia naturaleza, sociocultural e histórica, lo lúdico cuando es respetuoso, no impositivo y ético, torna fácil la transferencia de saberes a los contextos en que conviven las personas mayores y donde vivencian las más variadas relaciones sociales, para afirmar los procesos de re-definición de significados socioculturales acerca de la vejez y el envejecimiento, y favorece de modo natural influir en la transformación de las sedimentadas prácticas y representaciones tradicionales que modelan el sentir, el pensar y el hacer que todavía persisten en las comunidades acerca de vida en la vejez.

\section{Referencias}

COLECTIVO DE AUTORES. Las técnicas participativas y los juegos en la animación de grupos. In: _La animación sociocultural en la promoción de las personas adultas mayores. La Habana, Cuba: Experiencias de Cáritas Cubana, 2012. 
ELKONIN, D. B. Psicología del juego. La Habana, Cuba: Editorial Pueblo y Educación. 1984

RANGEL, C. L. L. Actividad pedagógica e interactividad en la educación de adultos mayores. In: CONVENCIÓN INTERNACIONAL "TECNOLOGÍA Y SALUD”, 2., 2014, La Habana, Cuba. Anais eletrônicos... La Habana: Memorias de la II Convención Internacional “Tecnología y Salud”, 2014. 1 CD-ROM.

. Procedimientos y herramientas interactivas: su incidencia en el desarrollo de los adultos mayores. Revista Electrónica Palabras Mayores, n. 8, mar. 2012. 